\title{
Tracking a temporal gap in band-limited noise: Frequency and level effects
}

\author{
PETER J. FITZGIBBONS \\ University of Maryland, College Park, Maryland
}

\begin{abstract}
Temporal gap resolution in band-limited noise signals was examined with a Békésy procedure in which listeners tracked the minimum signal intensity needed to resolve a periodic temporal gap of fixed duration. Gaps from $25 \mathrm{msec}$ to the smallest detectable value were tested to derive performance functions that described intensity threshold as a function of gap duration. Each of four octaveband signals, centered at .5, 1.0, 2.0 , and $4.0 \mathrm{kHz}$, was presented with a complementary filtered noise masker to restrict the listening band and avoid extraneous spectral cues. Performance functions were also derived in different levels of a white-noise background that was added to shift the subject's audibility thresholds to greater sound-pressure levels. Results show that gap resolution improves to a basic limit as signal level is increased from audibility threshold; the rate of improvement, however, increases progressively as audibility threshold is elevated. This observation suggests that shifts in audibility criteria for gap resolution are correlated with loudness changes. The smallest detectable gap for each signal was largely independent of sound-pressure level in a 35-40-dB range, but decreased systematically as a function of signal frequency. The frequency dependency is consistent with the hypothesis that temporal resolution is limited in part by spectral filtering in the auditory periphery.
\end{abstract}

Studies of temporal acuity in audition have for many years examined the ability of listeners to detect brief temporal intervals between successive stimulus events. According to Boring (1942), Exner, in 1875, reported that click stimuli needed to be separated by at least $2 \mathrm{msec}$ before listeners could begin to hear a succession of discrete sounds. Exner's value is not much different from current estimates of the threshold temporal gap between discrete bursts of broadband noise (Penner, 1975, 1977; Plomp, 1964). Only recently has it been observed that the ear's temporal resolving power exhibits an underlying spectral dependency (Fitzgibbons, 1983; Fitzgibbons \& Wightman, 1982; Shailer \& Moore, 1983). Those studies measured gap thresholds with various bandlimited noise stimuli, using procedures that avoided the problem of spectral confounding described in our earlier report (Fitzgibbons \& Wightman, 1982). The problem arises from the fact that temporal interruption of a narrowband signal causes a transient spread of energy to frequency regions outside the nominal signal band, thereby providing the listener with a salient spectral cue in gap detection. The artifact was avoided by use of filtered noise maskers to restrict the subject's listening band; the noise maskers were broadband, but had an attenuation band (spectral notch) introduced to enhance signal audibility at the

This research was supported in part by Biomedical Support Grants from PHS/NIH/DRR and grants from the General Research Board at the University of Maryland. The author's mailing address is: Department of Hearing and Speech Sciences, University of Maryland, College Park, MD 20742. desired test frequency. Collective results of the experiments revealed that gap threshold decreases progressively as the signal frequency is increased to about 5-6 kHz (8 kHz, Shailer \& Moore, 1983); at higher frequencies, measured to $14 \mathrm{kHz}$ (Fitzgibbons, 1984), gap threshold approaches an asymptote near 3 msec, close to Exner's original acuity estimate.

Results of the band-limiting experiments are theoretically interesting because they suggest that auditory temporal acuity may be limited by the same sensory mechanisms that impose a limit on auditory frequency resolution. Much of the psychophysical data on frequency resolution in audition are reasonably well described by a functional model of sensory processing that includes an initial stage of overlapping "critical band" filters, whose bandwidths become progressively wider at higher center frequencies (Fletcher, 1940; Patterson \& Green, 1978). In a physical linear system, the temporal resolving power of a filter is inversely proportional to its bandwidth in hertz. Thus, if critical-band filtering operates in a similar manner, we should expect psychophysical estimates of temporal resolution to reveal a systematic trend towards smaller thresholds with increased stimulus frequency. The empirical data are consistent with this hypothesis, at least through a broad lowfrequency range. With a broadband stimulus, it appears that subjects are able to listen selectively in high-frequency channels, in which widths of the peripheral filters are sufficient to permit optimal temporal resolution.

The present study extends the investigation of parameter dependencies in temporal acuity by 
examining the effects of stimulus intensity on gap resolution in different frequency bands. One motivation for examining level effects stems from consideration of the critical-band hypothesis. Critical bandwidths in audition have a number of operational definitions, but most of the classical empirical studies depict them as filter characteristics that are independent of signal intensity over a broad range above some minimum level (Scharf, 1970). However, some recent derived estimates of the auditory filter suggest that critical bands become progressively wider with increased signal level (Weber, 1977), perhaps more so in the higher frequency channels (Pick, 1980). On theoretical grounds, any significant level dependence in filter widths should produce a corresponding level dependence in temporal resolution.

The available narrowband data are generally consistent with the conclusion that gap threshold varies significantly with intensity at relatively low levels, but is largely independent of level for clearly audible signals. However, gap thresholds measured with adaptive forced-choice procedures were limited to two (Fitzgibbons \& Wightman, 1982) or three (Shailer \& Moore, 1983) fixed intensities, with the strongest level effects observed at low frequencies. This interaction may have been the consequence of the subjects' having elevated audibility thresholds at the lower frequencies. In another study (Fitzgibbons, 1983), we examined these low-level effects by reversing the experimental variables; subjects were asked to track the minimum signal level required to keep a periodic temporal gap of fixed duration at threshold. As expected, smaller gaps required greater intensity, but the smallest detectable gap was tracked at roughly equivalent relative intensities [about 35 -dB sensation level (SL)], independent of signal frequency in the decade .5-5 kHz. However, this outcome itself suggests that audibility criteria for gap detection may be better correlated with subjective intensity (i.e., loudness) than with stimulus intensity in decibels.

In the present experiment, we examine this assumption and the critical-band hypothesis, using the same tracking procedure with different band-limited noise signals. As before, each test signal is presented along with its complementary notched masker to restrict the listening band. Performance functions are derived by plotting intensity threshold as a function of gap duration. The procedure is then repeated with different levels of a white-noise background added to produce uniform shifts in the subject's audibility threshold at the test frequency. If intensity thresholds in gap detection are correlated with loudness changes, it should become evident in the performance functions, since loudness grows more rapidly with elevated audibility thresholds (e.g., Stevens \& Guirao, 1967). The white-noise background will also require subjects to track at progressively greater sound-pressure levels, allowing us to determine whether or not the basic limits in temporal resolution are level dependent.

\section{METHOD}

\section{Subjects}

Three subjects with normal pure-tone sensitivity (re: ANSI S3.61969) participated in the experiment. None of the subjects had had prior experience in psychoacoustic tasks, and each received several hours of practice prior to data collection. The subjects listened monaurally in a sound-treated booth in 1-2-h sessions over the course of 3-4 weeks.

\section{Stimuli}

The stimuli were four continuous octaveband noises, centered at $.5,1,2$, and $4 \mathrm{kHz}$, with off-band attenuation rates of $96 \mathrm{~dB} /$ octave. Each signal was fed through an electronic switch that was triggered periodically to produce a temporal gap at the rate of $1 \mathrm{~Hz}$; waveforms at each gap were shaped by a l-msec cosine risefall envelope, with gap duration defined as the interval between 3dB down points the envelope. The notched masker for each signal was generated by passing a white noise through two filters (Krohn-Hite 3343) arranged to operate in a band-stop mode. The filters were set to produce an octave-wide attenuation band centered at the signal center frequency; slopes within the notch were $96 \mathrm{~dB} /$ octave, with a notch depth of $45 \mathrm{~dB}$ at each center frequency. The signal and masker were added, with the spectrum level of the masker outside the notch set $20 \mathrm{~dB}$ below the spectrum level of the signal at its center frequency. This masker setting was sufficient to eliminate off-band spectral cues, as determined previously (Fitzgibbons, 1983). All waveform and spectral characteristics of signals and maskers were examined with the aid of a digital storage oscilloscope and FFT-computing spectrum analyzer.

\section{Procedure}

The signal-masker combination provided the input to a Békésytype tracking attenuator, the output of which was delivered to a single TDH-49 earphone with MX41/AR cushion. The attenuator characteristics (linearity and frequency response) preserved the input signal-to-masker ratio at the output throughout its $100-\mathrm{dB}$ operating range. With each signal, the duration of the periodic gap was preset, and subjects tracked the minimum signal intensity needed to keep the gap audible. For each of several gap values tested in this manner, the average of about 25 midpoints in the updown level excursions was taken as a trial-run estimate of intensity threshold. Successive trial-run estimates were collected until the most recent three fell within a range of $5 \mathrm{~dB}$; a mean of these three trial-run estimates was then recorded as a final estimate of intensity threshold for the particular gap value. Gap durations were tested in descending order using values of 25,15 , and $10 \mathrm{msec}$, followed in turn by values decreased in 2 -msec steps until the subject failed to track a final threshold estimate. At this point, additional gap values were tested by progressively halving the previous 2 -msec step size to determine the minimum detectable gap to the nearest .25 msec. Overall, the procedure produced four to six data points per signal to construct the performance curves showing intensity threshold as a function of gap duration.

In the same manner, a performance curve for each signal was derived in each of three levels of a white-noise background that was added to the signal-masker combination at the earphone terminals. The overall levels of the white noise, measured in a 6-cc coupler, were 50, 70, and $85 \mathrm{~dB}$ sound-pressure level (SPL). Audibility thresholds in decibels SPL were also measured for each signal, in quiet and at each level of white-noise background. For these measurements, the octaveband signal, presented continuously and without the notched masker, was fed through the attenuator, and subjects tracked a detection threshold in the same manner as described above. It should be noted also that the notched maskers do not limit signal audibility within the nominal octavebands, and 
thus have no important influence on intensity thresholds recorded in the experiment.

\section{RESULTS}

The experiment produced orderly results, which are displayed in the separate quadrants of Figure 1. The ordinate of the figure plots final estimates of the threshold signal level in decibel sensation level (re: signal audibility threshold), with gap duration in milliseconds scaled logarithmically along the abscissa. Numbers in the upper left of each quadrant give the SPL of the white-noise background, with 0 referring to its absence. The parameter associated with the performance curves is the center frequency in kilohertz of the octaveband signal. Data points on each curve are mean thresholds of the three subjects; individual deviations were relatively small, being within $5 \mathrm{~dB}$ for each intensity threshold displayed. The horizontal coordinate of the leftmost point on each curve is a mean of the subjects' minimum detectable gap values; deviations in this temporal threshold were also small, and never exceeded $1.5 \mathrm{msec}$.

Performance curves derived without the whitenoise background (upper left quadrant, Figure 1) reveal the same trends that were reported earlier (Fitzgibbons, 1983) for somewhat higher frequency noise

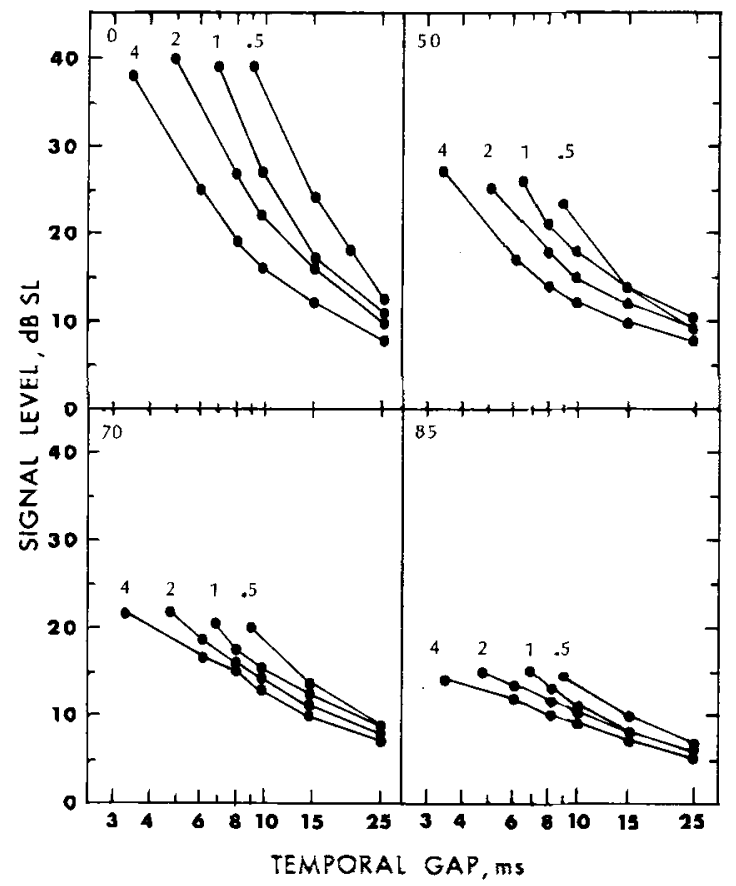

Figure 1. Threshold signal level in decibels senantion level (SL) as a function of temporal gap durntion in milliseconds on a log. arthmic seale. Numbers in the upper left of each quadrant represent the white-noise background level in decibels sound-pressure level (SPL) (overall), with 0 referring to the condition of its absence. The parameter associated with the performance curves is the octaveband center frequency in kilohertz. Data points are means of three subjects.
Table 1

Mean Audibility Thresholds of Subjects for Each Octaveband Signal and Background Noise Level

\begin{tabular}{ccrrrr}
\hline & \multicolumn{5}{c}{ Octaveband Center Frequency in kHz } \\
\cline { 2 - 6 } $\begin{array}{c}\text { Background } \\
\text { Level }\end{array}$ & \multicolumn{1}{c}{5} & 1.0 & 2.0 & \multicolumn{1}{c}{4.0} & \multicolumn{1}{c}{ Mean* } \\
\hline 0 & 11.5 & 8.0 & 6.0 & 9.0 & 8.6 \\
50 & 32.3 & 35.0 & 35.3 & 38.3 & 35.2 \\
70 & 53.7 & 55.7 & 55.3 & 59.0 & 55.9 \\
85 & 69.0 & 70.3 & 69.7 & 73.7 & 70.7 \\
\hline
\end{tabular}

Note-Entries are overall sound pressure levels in $\mathrm{dB}$ re: 20 micropascals. *Averaged across signal frequency.

bands. Each curve shows that the resolution of progressively smaller gaps required an increase in signal level, with the smallest detectable gap tracked at approximately the same relative intensity for each signal. The influence of signal frequency is exhibited by the shifts in the performance function to smaller gap values with increased frequency. The curves also tend to converge at low levels, revealing that the strength of the spectral dependency diminishes near audibility threshold. The other quadrants of Figure 1 display the same general trends, except that the performance curves are compressed to a progressively narrower intensity range as the background noise level is increased.

The data on audibility thresholds for each signal and background noise level are presented in Table 1. The table entries are mean values in decibels SPL (overall) for the three subjects; individual thresholds fell within a range of 2-3 dB in each case. Averaged across signal frequency, audibility thresholds shifted from 8.6 to $70.7 \mathrm{~dB}$ SPL through the range of background levels employed in the experiment. Table 2 provides numerical values of the minimum detectable gaps in milliseconds, as displayed by the leftmost point on the performance curves. Also shown in Table 2 (rightmost columns) are the mean intensity thresholds in decibels SPL and decibels SL (averaged across frequency) associated with the minimum gaps in each level of background noise; these intensity thresholds increased from 47.9 to $85.3 \mathrm{~dB}$ SPL, but decreased from 39.3 to $14.6 \mathrm{~dB}$ SL with progressive elevation of audibility thresholds. The table also reveals that the minimum gap values showed little, if any, level dependence, but a strong frequency dependency. A least squares straight line fit to the mean gap threshold (across levels) as a function of log signal frequency has a slope of $6.43 \mathrm{msec} / \mathrm{log} \mathrm{Hz}$ (1.93 msec/octave), with a product-moment correlation (r) of .99. Individual straight-line fits to the data for each background level gave nearly identical results.

\section{DISCUSSION}

The experiment reveals that auditory temporal resolution has a strong level dependency, but only 
Table 2

Mean Gap Thresholds (in Milliseconds) of Subjects for Each Octaveband Signal and Background Noise Level

\begin{tabular}{|c|c|c|c|c|c|c|c|c|}
\hline \multirow{3}{*}{$\begin{array}{c}\text { Background } \\
\text { Level }\end{array}$} & \multicolumn{4}{|c|}{ Gap Thresholds } & & & & \\
\hline & \multicolumn{4}{|c|}{ Center Frequency in $\mathrm{kHz}$} & \multicolumn{4}{|c|}{ Intensity Thresholds* } \\
\hline & .5 & 1.0 & 2.0 & 4.0 & Mean SPL & SD & Mean SL & SD \\
\hline 0 & 9.2 & 7.0 & 5.0 & 3.3 & 47.9 & 1.8 & 39.3 & 1.0 \\
\hline 50 & 9.1 & 6.8 & 4.9 & 3.4 & 60.3 & 4.1 & 25.1 & 1.8 \\
\hline 70 & 9.0 & 6.8 & 4.8 & 3.3 & 76.4 & 3.4 & 20.5 & 1.6 \\
\hline 85 & 9.2 & 6.7 & 4.8 & 3.4 & 85.3 & 1.6 & 14.6 & .53 \\
\hline Mean & 9.1 & 6.8 & 4.9 & 3.3 & & & & \\
\hline SD & .10 & .13 & .10 & .06 & & & & \\
\hline
\end{tabular}

Note-Background levels are overall sound pressure levels in $\mathrm{dB}$ re: 20 micropascals. $\quad$ *On the right are means and standard deviations of intensity thresholds (in $d B S P L$ and $d B S L$ ), averaged across signal frequency, at the minimum gaps.

within a limited range above audibility threshold. Within this range, the sensation level required for optimal resolution varies inversely with audibility threshold. We can infer from this finding that the intensity criteria for gap resolution are closely correlated with subjective magnitude of the stimulus. It is well known that the partially masked normalhearing listener experiences loudness recruitment, which is manifested as an increase in the initial rate of loudness growth above audibility threshold as the threshold is elevated (Hellman \& Zwislocki, 1964; Stevens \& Guirao, 1967). The corresponding decrease in the linear-component slopes of the derived performance curves is consistent with these loudness changes. The recruitment-like effects in the performance curves appear to be relatively uniform across signal frequency, except for the somewhat greater initial compression of the $500-\mathrm{Hz}$ curve (upper right quadrant, Figure 1); this exception may simply reflect the more rapid growth of loudness that occurs below $1 \mathrm{kHz}$ (Scharf, 1978).

In addition to the relative level effects, the limits of temporal resolution were found to be essentially independent of sound pressure level over a 35-40-dB range. This was true for each of the frequency bands tested. With regard to the critical-band hypothesis, the results are in agreement with the conclusion that widths of the peripheral auditory filters remain invariant with changes in signal level over a substantial range. With our low-level data (Fitzgibbons, 1983), we demonstrated that gap thresholds in the range $.5-5 \mathrm{kHz}$ were linearly related to changes in the critical band (e.g., Scharf, 1970) through the same frequency range. Shailer and Moore's (1983) data, also collected at a moderate intensity (25-dB spectrum level), reveal a similar relationship through a somewhat broader range $(.2-8 \mathrm{kHz})$. Present results indicate that this correlation between temporal and frequency analysis in audition does not depend heavily on stimulus level.

Despite these strong correlations in the data, it does not appear that simple estimates of auditory filter widths can be obtained through gap-threshold measurements. For example, if the bandwidth of a signal is progressively increased about a given center frequency, one might expect to observe a decrease in gap threshold once the bandwidth exceeds some critical value, that is, once stimulus energy spills into the passband of the adjacent higher frequency filter with its broader width. In our earlier experiment (Fitzgibbons, 1983), we varied signal bandwidth over a 2 octave range at different center frequencies, but found no significant shift in gap threshold. Shailer and Moore (1983) did observe a decrease in gap threshold with increased signal bandwidth, particularly at low center frequencies with bandwidths less than $200 \mathrm{~Hz}$ or so. However, the change in gap threshold was progressive, with no clear breakpoint that could signify the existence of a critical bandwidth as such. Incidentally, the bandwidth data provide little support for an alternative argument that subjects can simply adjust the width of some internal filter to accommodate the signal's passband, as a strategy to optimize temporal resolution.

Another logical approach towards examining filter widths that operate in temporal resolution would be to measure the threshold gap between signals that differ in frequency. For signals close in frequency, it is usually presumed that gap detection is limited by a short-term sensory persistence of the leading signal. It would seem to follow that signals delivered to different peripheral filters would interact less, produce less sensory "filling in" of the gap, and thus allow better temporal resolution. Williams and Perrott (1972) found the opposite effect; they used pairs of tone bursts and observed an increase in gap threshold with increased tonal frequency separation. Later, Williams and Elfner (1976) used three-tone sequences and reported that gap threshold at first increases with frequency separation, but then decreases once signals bordering the gap are separated by more than a critical band. This latter finding is interesting, but it does not explain the initial increase in gap threshold, or the lack of a similar critical-band effect with twotone sequences.

Clearly, simple descriptive models of sensory pro- 
cessing do not provide an adequate account of each parameter dependency observed in temporal resolution. Nor is it reasonable to expect that discrimination cues in each temporal resolution task and stimulus environment derive solely from the auditory periphery. However, for stimuli used in the present experiment, it appears that temporal resolution may be limited at an early stage of sensory coding, one that is more commonly associated with performing a spectral analysis of stimulus events. Our results also indicate that the audibility levels required for optimal temporal resolution are probably best described as subjective criteria.

The latter observation has other implications, particularly with regard to the interpretation of temporalacuity data collected from listeners with sensorineural hearing impairment (e.g., Fitzgibbons \& Wightman, 1982). For gap detection, like all suprathreshold tasks, performance comparisons between normal and hearing-impaired subjects can be made with stimulation equated for SPL or SL, the choice not always being obvious. Subjects with cochlear damage typically exhibit elevated audibility thresholds and loudness recruitment, factors shown here to have an important influence on temporal resolution. This evidence suggests that the appropriate choice of comparison intensity for study of temporal acuity in hearing-impaired listeners would be sound-pressure level rather than sensation level.

\section{RETERENCES}

ANSI (1969). Specifications for audiometers (ANSI S3.6-1969). New York: American National Standards Institute.

Boring, E. G. (1942). History of sensation and perception in experimental psychology. New York: Appleton-Century-Crofts.

Fitzoibbons, P. J. (1983). Temporal gap detection in noise as a function of frequency, bandwidth, and level. Journal of the Acoustical Society of America, 74, 67-72.

Fitzorbbons, P. J. (1984). Temporal gap resolution in narrowband noises with center frequencies from $6000-14000 \mathrm{~Hz}$. Journal of the Acoustical Society of America, 75, 566-569.
Fitzaibions, P. J., \& Wightman, F. L. (1982). Gap detection in normal and hearing-impaired listeners. Journal of the Acoustical Society of America, 72, 761-765.

Fletchen, H. (1940). Auditory patterns. Reviews of Modern Physics, 12, 47-65.

Hellman, R. P., \& Zwiglocki, J. J. (1964). Loudness function of a 1000-cps tone in the presence of a masking noise. Journal of the Acoustical Society of America, 36, 1618-1627.

Patterson, R. D., \& Green, D. M. (1978). Auditory masking. In E. C. Carterette \& M. P. Friedman (Eds.), Handbook of perception: Vol. 4. Hearing (pp. 337-359). New York: Academic Press.

Penner, M. J. (1975). Persistence and integration: Two consequences of a sliding integrator. Perception \& Psychophysics, 18, 114-120.

Penne R, M. J. (1977). Detection of temporal gaps in noise as a measure of the decay of auditory sensation. Journal of the Acoustical Society of America, 61, 552-557.

PICk, G. F. (1980). Level dependence of psychophysical frequency resolution and auditory filter shape. Journal of the Acoustical Society of America, 68, 1085-1095.

Plomp, R. (1964). Rate of decay of auditory sensation. Journal of the Acoustical Society of America, 36, 277-282.

Schanf, B. (1970). Critical bands. In J. V. Tobias (Ed.), Foundations of modern auditory theory (pp. 157-202). New York: Academic Press.

ScharF, B. (1978). Loudness. In E. C. Carterette \& M. P. Friedman (Eds.), Handbook of perception: Vol. 4. Hearing (pp. 187-242). New York: Academic Press.

Shailer, M. J., \& Moore, B. C. J. (1983). Gap detection as a function of frequency, bandwidth, and level. Journal of the Acoustical Society of America, 74, 467-473.

Stevens, S. S., \& Guirao, M. (1967). Loudness functions under inhibition. Perception \& Psychophysics, 2, 459-465.

Weber, D. L. (1977). Growth of masking and the auditory filter. Journal of the Acoustical Society of America, 62, 424-429.

Williams, K. N., \& ElfNen, L. F. (1976). Gap detection with three auditory events-a single-channel process. Journal of the Acoustical Society of America, 60, 423-428.

Williams, K. N., \& Perrotr, D. R. (1972). Temporal resolution of tonal pulses. Journal of the Acoustical Society of America, 51, 644-647.

(Manuscript received August 23, 1983; revision accepted for publication March 22, 1984.) 